\title{
Diagnostic Test of Chest Radiograph on Fungal Pneumoniae
}

\author{
Ana Majdawati ${ }^{1}$ Inayati $^{2, *}$ \\ ${ }^{1}$ Radiology Department of Medical Study Program Faculty of Medicine and Health Sciences Universitas Muhammadiyah \\ Yogyakarta. Indonesia \\ Email: anamjdwt2@gmail.com \\ ${ }^{2}$ Microbiology Department of Medical Study Program Faculty of Medicine and Health Sciences Universitas Muhammadiyah \\ Yogyakarta, Indonesia \\ *Corresponding Author. Email: inaythabib@yahoo.co.id
}

\begin{abstract}
Fungal pneumoniae is lung infection caused by fungi in which clinical manifestations are not typical, even asymptomatic. Fungal pneumonia prevalences are $68.3 \%$ in both lung tuberculosis and non-tuberculosis. Fungal pneumoniae is often undiagnosed early but later or late diagnosed and it make fails or not optimal treatment. Clinical manifestation and radiograph Fungal pneumoniae are not specific. The aim of this study is to know sensitivity and spesificity of chest radiograph on Fungal pneumoniae. Fifty five suspected Fungal pneumoniae patients are examined using the chest radiograph (homogeneous round or infiltrate presentation, cavity, compaction of hilum lymphonody in the form of evenly calcified miliary lesions, abscess images) and sputum Fungal culture to determine sensitivity and specificity using Diagnostic test. Positive Fungal culture are $21,8 \%$ smaller than Negative Fungal culture (78.2\%). The sensitivity value is $83.3 \%$ and a specificity value is $25.6 \%$. In conclusion chest radiograph can be used as a screening examination in patients with suspected Fungal pneumoniae, However, it cannot be used as a screening for healthy patients which showed a negative Fungal culture while the patients are suspected Fungal pneumoniae.
\end{abstract}

Keywords: Fungal pneumoniae, sensitivity, specificity, chest radiograph, sputum Fungal culture

\section{INTRODUCTION}

Pulmonary Fungal infections (Fungal pneumonia) are lung infections caused by fungi. Most Fungal pneumonia are caused by Candida albicans (70\%) followed by Aspergillosis (22\%) and Mucormycosis (8\%) with a higher prevalence of men than women 4: 1 [1]. Clinical symptoms of specific Fungal pneumonia is not typical, asymptomatic, symptoms are mild and patients are unaware of being infected by fungi [2]. Some patients suffer from severe illness with symptoms of prolonged coughing and shortness of breath wich cannot be improved with treatment do not improve with treatment. The occurrence of Fungal pneumonia in patients with risk factors such as malignancy, Chronic Obstructive Pulmonary Disease (COPD), Diabetes Mellitus (DM), HIV AIDS, Tuberculosis, and pulmonary infectious caused by pyogenic bacteria, immunocompromised disease and long-term corticosteroid use. Fungal pneumonia is often undiagnosed early, because clinical symptoms are not typical. Fungal pneumonia is sometimes overlooked by doctors, due to the major diseases or risk factors that become a concern by doctors for their treatment $[1,3,4]$. This Fungal infection can reach $68.3 \%$ both in pulmonary tuberculosis and non-pulmonary tuberculosis [1]. Definitive diagnosis requires examination of culture and serology that requires much time and high costs [5].

Chest radiograph is still often done in Indonesia as a preliminary examination of suspected Fungal pneumonia, although some chest radiograph images of Fungal pneumonia are similar to tuberculosis or non-specific bacterial infections [6]. Chest radiograph examination Fungal pneumonia in several developed countries has been abandoned. They switched to superior modalities such as Chest Computed Tomography (Chest CT scan), Magnetic Resonance Imaging (MRI) and Ultrasonography (USG) [7]. This examination is still rarely done in Indonesia given the high cost and not all health service places have these modalities. The role of chest radiograph is still considered important because it is fast, inexpensive and easy to screen for lung infections due to fungi because there are some typical radiological lesions for certain types of Fungal pneumonia [1,6]. Some chest radiograph manifestations of Fungal pneumonia, including: unilateral or bilateral segmental consolidation with or without cavity, nodular infiltrate, "target meniscus", pleural thickening, compaction of hilar lymph nodes, homogeneous consolidation of round forms in the hilar region resembling Bronchogenic carcinoma, scattered nodular lesions in both lungs, calcification, the lesions similar to pulmonary tuberculosis (milliard, infiltrate) [6-9].

Several reasons as stated above, caused the authors to carry out research which aimed to obtain the value and sensitivity and specificity of chest radiograph in Fungal pneumonia in general, without grouping species. The results of this sensitivity and specificity can be used as a consideration for reference to diagnosis or initial screening of suspected Fungal pneumonia. Good diagnostic test results can be used as a reference for a quick diagnosis and therapy can be given immediately. 


\section{LITERATURE REVIEW}

\subsection{Fungal pneumonia}

Fungal pneumonia is still relatively rare when compared to bacterial or viral pneumonia. Important initial diagnosis is immediately enforced because it can reduce morbidity and mortality [4]. There are 3 types of fungi that often cause pneumonia [9,11], namely Histoplasma capsulatum causes histoplasmosis; Coccidioides immitis causes coccidiomycosis; Blastomyces dermatitidis causes blastomycosis. Other Fungal infections often occur in patients with immune system disorders (immunocompromise), namely: Cryptococcosis caused by Cryptococcus neoformans. Cryptococcosis is most commonly found, can occur in people who are healthy but usually will be severe if it afflicts patients with immune system disorders such as AIDS. Cryptococcosis can spread, especially to the meningens, which will cause Cryptococcal meningitis. Aspergillosis caused by Aspergillus Sp. Aspergillus causes lung infection in people with AIDS or someone who is undergoing an organ transplant. Candidiasis caused by Candida Sp. Infrequent infections, namely pulmonary candidiasis (bronchial and pulmonary candidiasis), often occur in leukemias undergoing chemotherapy. Mukormikosis is a rare Fungal infection, often affecting people with severe Diabetes or Leukemia.

\subsection{Diagnosis of Fungal pneumonia}

\subsubsection{Clinical symptoms}

The diagnosis of Fungal pneumonia is very difficult because most are asymptomatic and often like regular flu symptoms or pulmonary infections by other causes. Clinical symptoms are not typical and difficult to distinguish from other microorganism infections such as pneumonia due to bacteria, viruses, parasites. Suspicion of Fungal pneumonia, if there is a presdipositioning factor, is immediately followed up by examination of the specimen so that the diagnosis can be immediately determined. Diagnosis Fungal pneumonia is established based on history (fever with temperatures over 400C, chills, coughing with mucoid or purulent phlegm sometimes accompanied by blood, shortness of breath and chest pain). High risk factors for Fungal pneumonia, ie symptoms of pneumonia do not diminish with administration of antibiotics [10].

\subsection{Supportive diagnosis}

\subsubsection{Radiology examination}

Chest radiograph have an important role in diagnosing Fungal pneumonia, although sometimes there are some features similar to those of tuberculosis or non-specific bacterial infections. Some chest radiograph of Fungal pneumonia, including [7,8,11-13]:

a) Pulmonary aspergillosis: homogeneous, evenly consolidated or small circular shadows with irregular nodular infiltrates very similar to pulmonary tuberculosis, and sometimes miliary spread. General radiological features of opportunistic pulmonary aspergillosis are round cavity lesions with "air sickle" nodules, "meniscus targets", "bull eyes" or penises. Sometimes enlargement of the hilum lymph is found.

b) Blastomycosis: homogeneous consolidation, round shape in the hilum region and resembling Bronkhogenic Carcinoma

c) Histoplasmosis: nodular lesions spread in both lungs, calcification (+), compaction of the hilum lymphnode, pleural involvement can occur and a tuberculous lesion similar to the lung

d) Kokisiiodomikosis: miliary lesions spread in the lung, like tuberculous billion. Sometimes images of cavities are found with infiltrates around them

\subsubsection{Microbiology examination}

Positive Fungal pneumonia if the fungus can be proven, either through direct examination or culture. Examination of sputum: microscopic fungi and culture; bronchoscopy: bronchial secretions, bronchial rinses, transbronchial pulmonary biopsies; pulmonary aspiration with needles; blood laboratory examination; blood culture; Serological examination. Definitive diagnosis requires culture and serological examination $[4,5,14]$.

\section{CONCEPTUAL FRAMEWORK}

\begin{tabular}{|c|c|}
\hline Chest radiograph suspected of & \\
\hline $\begin{array}{l}\text { Fungal pneumonia } \\
\text { Positive chest radiograph: } \\
\text { - Unilateral / bilateral consolidation } \\
\text { - Cavity with nodules "air cresent" } \\
\text { (target meniscus), fungus ball } \\
\text { - Homogeneous round lesions, in } \\
\text { the hilum area with calcification } \\
\text { - Hilar lymphnode in the both } \\
\text { lungenlarges } \\
\text { - Millennial nodules, spread evenly }\end{array}$ & $\begin{array}{l}\text { Results of sputum } \\
\text { culture in Sabarouth } \\
\text { Agar media: } \\
\text { Positive fungus: } \\
\text { the result of culture is } \\
\text { fungal growth } \\
\text { Negative fungus: } \\
\text { the results of culture } \\
\text { have no fungal growth }\end{array}$ \\
\hline $\begin{array}{l}\text { Negative chest radiograph: } \\
\text { Normal pulmo, no lesions in the } \\
\text { both lung }\end{array}$ & \\
\hline
\end{tabular}

Figure 1. Conceptual Framework Scheme

\section{RESEARCH METHOD}

The method of this study is a diagnostic test, determine the value of sensitivity and specificity of chest radiograph images in patients suspected of Fungal pneumonia with a gold standard of sputum culture in Sabarouth Agar media with $2 \times 2$ tables.

The study sample was a patient's sputum suspected of Fungal pneumonia that met the inclusion and exclusion criteria. The inclusion criteria are patients aged 15-60 years, clinical symptoms (at least there are symptoms of cough and fever). Criteria for fever are fever accompanied by shivering or not, body temperature over $38^{0} \mathrm{C}$; one of the symptoms of cough: mucoid / purulent cough, shortness of breath can be accompanied by chest pain or not and the history of chronic diseases. Correct taking of sputum, which is asking the patient to rinse first. If only sputum is desired, the best sputum is the morning sputum which is accommodated in a wide and sterile mouth container such as a petri dish, a wide mouthed bottle, 
sputum cardboard etc. sputum must always be seen as infectious material. Work desk and microscope should be sterilized with Lysol 10\%. The research sample can come from the polyclinic and inpatient care, the period of 2016/2017 in PKU Muhammadiyah Hospital Yogyakarta and PKU Muhammadiyah Gamping Hospital, Yogyakarta.

\subsection{Work procedures}

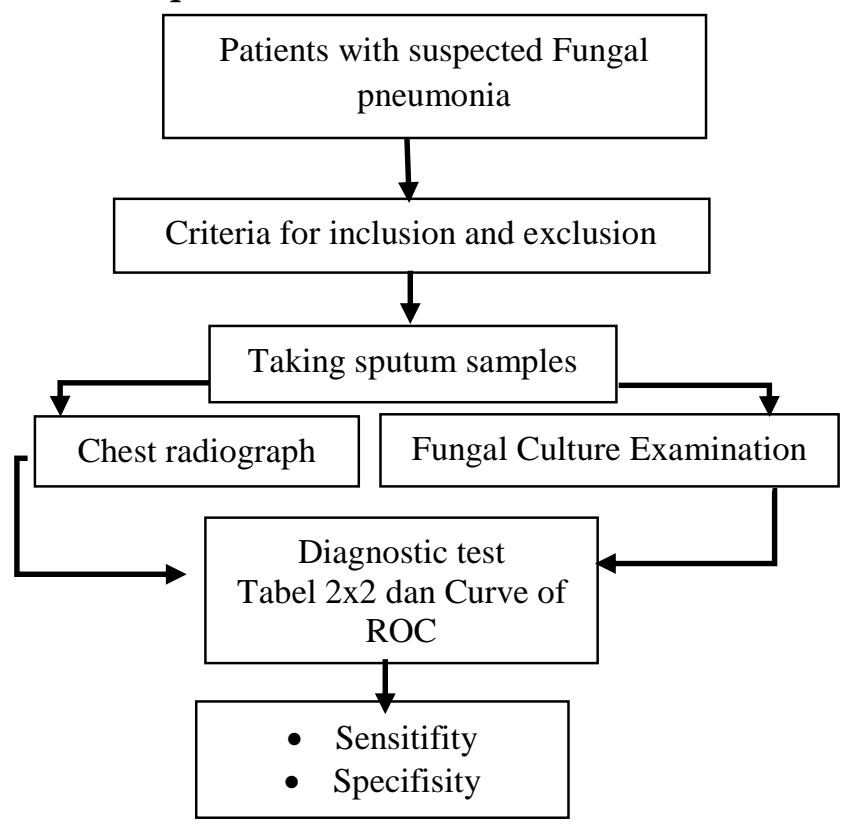

Figure 2. Work procedures

\subsection{Research Variables}

Independent variable:

- Chest radiograph suspected of Fungal pneumonia, positive results

- Chest radiograph suspected of Fungal pneumonia, negative results

Dependent variable

a) Results of Suspect patient's sputum culture Fungal pneumonia, positive culture

b) Results of sputum culture of patients suspected of Fungal pneumonia, negative culture

\subsection{Operational Definition of Variables}

a) Chest radiograph suspected of Fungal pneumonia positive results: if chest radiograph results are at least 1 of the images: unilateral / bilateral consolidation; cavity with nodules "air cresent" (target meniscus), fungus ball; homogeneous round lesions in the hilum region with calcification; enlarged hilar lymphnode; miliary nodules are spread evenly.

b) Chest radiograph are suspected of Fungal pneumonia, negative results: if the chest radiograph is not obtained lesions or abnormalities in both the lungs and heart and bone schema (normal chest radiograph)

c) The results of the patient's sputum culture suspect positive Fungal pneumonia: if on the medium of Fungal growth (Sabarouth agar) planted by sputum the patient suspects Fungal pneumonia overgrown with fungus

d) Suspect culture results of the patient's sputum Negative Fungal pneumonia: if the Fungal growth media
(Sabarouth agar) is planted by the patient's sputum is suspected Fungal pneumonia is not overgrown with fungi

\section{RESULTS AND STATISTICAL ANALYSIS}

\subsection{Results}

Diagnostic test studies of chest radiograph of suspected patient's Fungal pneumonia involving 110 research subjects with clinical symptoms leads to a diagnosis of suspected Fungal pneumonia. Characteristics of research subjects as shown in table.

TABLE 1. ChaRACTERISTICS OF SUSPECTED SUBJECTS WITH Fungal PNEUMONIA BASED ON AGE, CLINICAL SYMPTOMS AND DURATION OF CLINICAL SYMPTOMS.

\begin{tabular}{|c|c|c|c|}
\hline No & Description & Frequency & Percentage \\
\hline \multirow[t]{3}{*}{1} & Age & & \\
\hline & Adult $(<50$ tahun $)$ & 48 & 43,6 \\
\hline & Eldery ( $\geq 50$ tahun $)$ & 62 & 56,4 \\
\hline \multirow[t]{4}{*}{2} & Clinical Symptoms & & \\
\hline & Cough, shortness of breath $+/-$ (mild) & 78 & 70,9 \\
\hline & $\begin{array}{l}\text { Cough, shortness of breath, fever } \\
\text { (moderate) }\end{array}$ & 12 & 10,9 \\
\hline & $\begin{array}{l}\text { Cough }+ \text { blood, shortness of breath, chest } \\
\text { pain, etc. (Weight) }\end{array}$ & 20 & 18,2 \\
\hline \multirow[t]{3}{*}{3} & Duration of clinical symptoms & & \\
\hline & Less 2 weeks & 38 & 34,6 \\
\hline & Over 2 weeks & 72 & 65,4 \\
\hline
\end{tabular}

Study subjects in the age group $>50$ years showed more than $<50$ years of age. This shows that the age of $>50$ years has decreased the immune system and has a higher risk of Fungal pneumonia.

Clinical symptoms found in research subjects varied. Clinical symptoms include coughing, coughing up blood, shortness of breath, fever and chest pain. Existing clinical symptoms can be grouped according to 3 categories namely mild, moderate clinical symptoms and severe clinical symptoms as shown in table 1. The majority of the study subjects showed mild clinical symptoms, namely cough and shortness of breath as much as $70.9 \%$, followed by severe clinical symptoms of cough with blood, shortness of breath, chest pain and other symptoms as much as $18.2 \%$ and moderate clinical symptoms of coughing, shortness of breath and fever as much as $10.9 \%$. The duration of clinical symptoms in the study subjects mainly occurred more than 2 weeks as much as $65.4 \%$.

The subjects of the study were chest radiograph examination and examination of Fungal cultures from sputum specimens. The results of chest radiograph examination are shown in figure 1 below. 


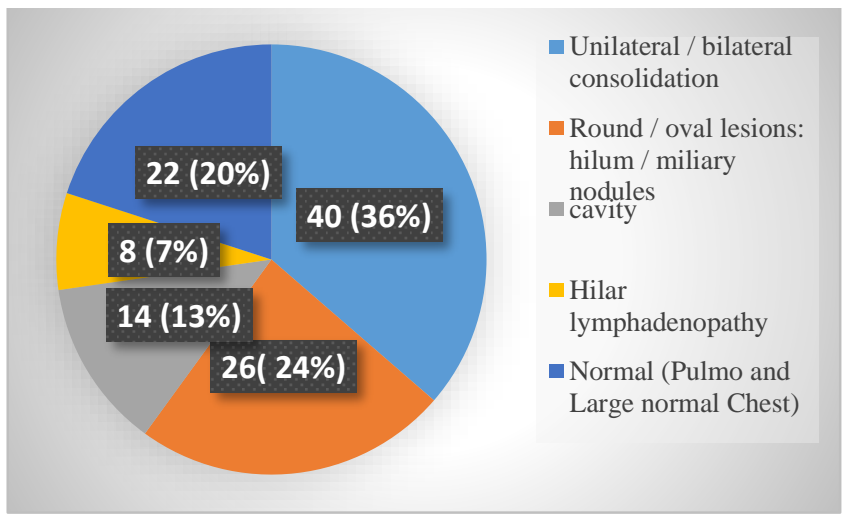

Figure 3. Overview of lesions on chest radiograph research subjects

Figure 4,5,6 shows clinical patients' Fungal pneumonia with positive Fungal culture results. Some radiological features are the presence of milliary nodules, hilar lymphadenopathy, cavity. The majority of study subjects showed abnormal chest radiograph of as much as $80 \%$ and research subjects who showed normal chest radiograph of as much as $20 \%$ as shown in figure 4.

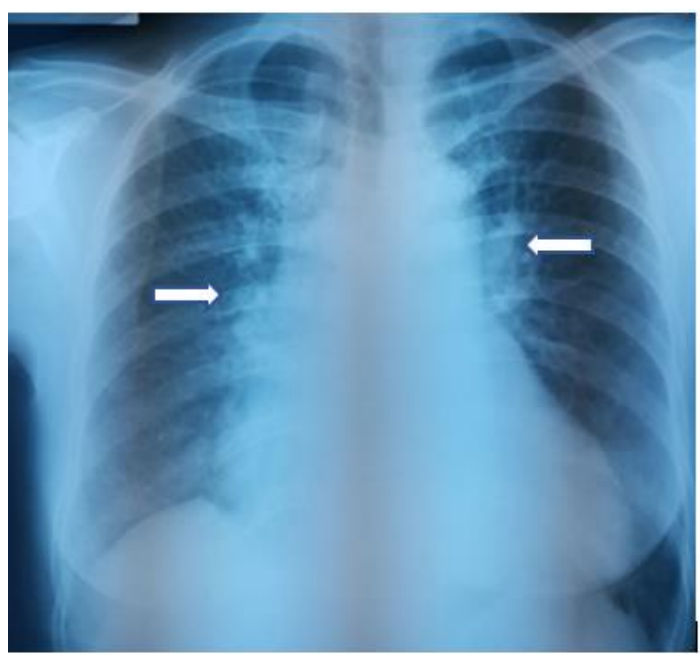

Figure 4. Chest radiograph patients with Fungal pneumonia, hilar lymphadenopathy (white arrow)

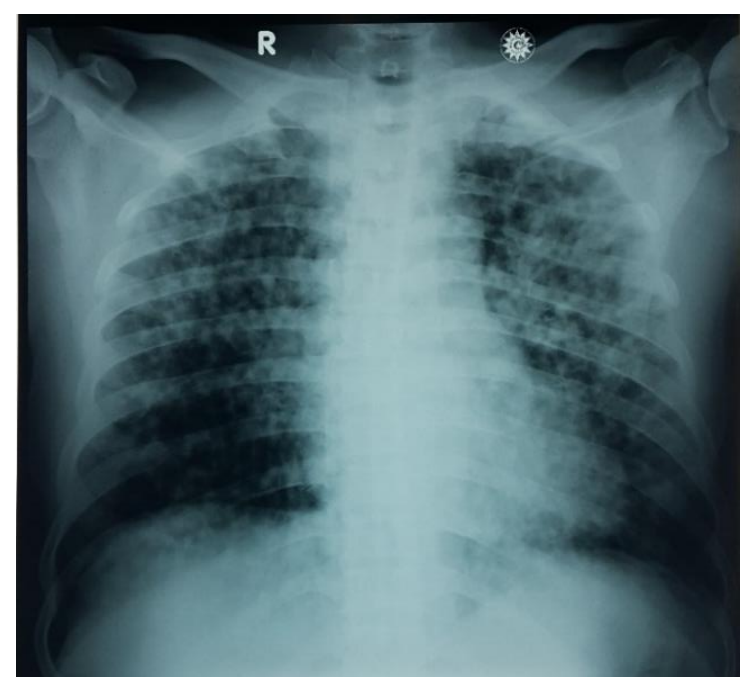

Figure 5. Chest radiograph patient with Fungal pneumonia, milliary nodules in both lung

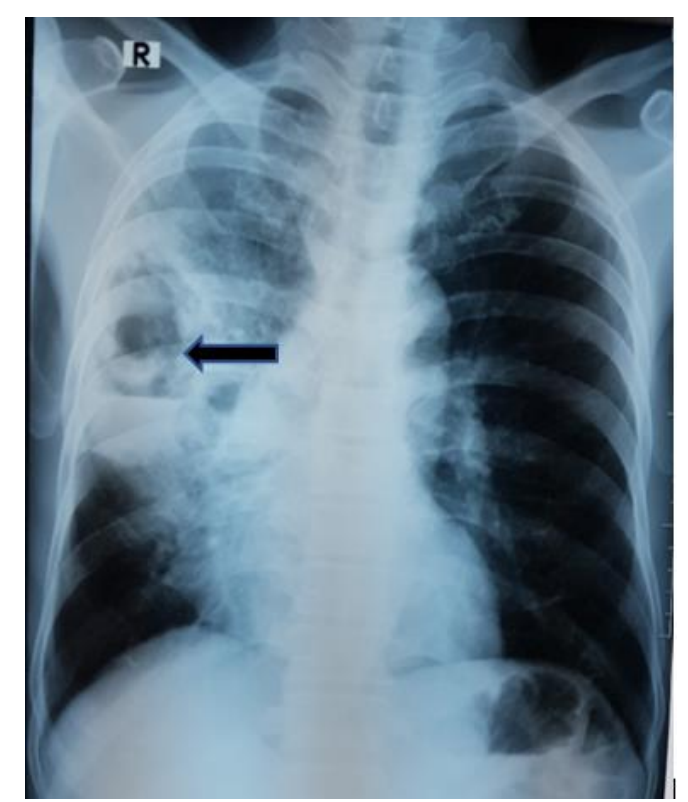

Figure 6. Chest radiograph patient with Fungal pneumonia, Cavity (black arrow)

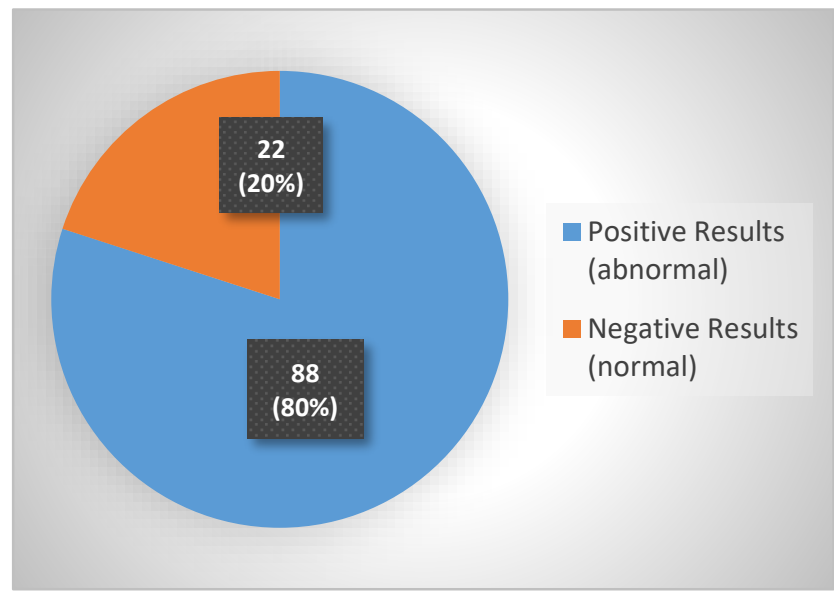

Figure 7. Results of chest radiograph examination of the research subjects

The results of the examination of positive Fungal cultures where Fungal growth was found on the examination of sputum specimens in the study subjects were $22 \%$ while the results of negative Fungal culture examination where no Fungal growth was found showed a greater number of $78 \%$ as shown in figure 8. 


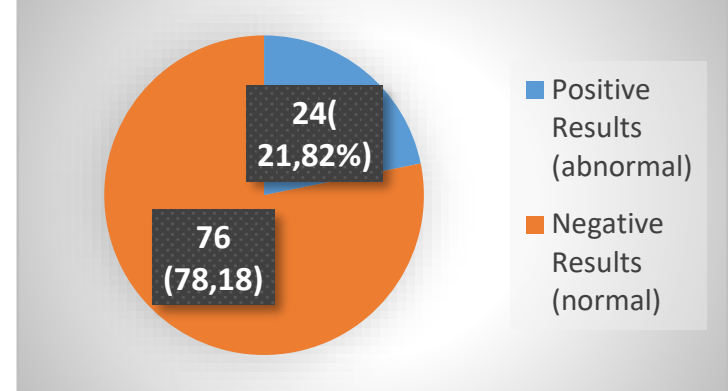

Figure 8. The results of examination of the Fungal culture of the research subjects

The results of chest radiograph and Fungal cultures on graphs 2 and 3, calculated sensitivity and specificity with a 2x2 table. Diagnostic results of chest radiograph tests in suspected research subject's Fungal pneumonia with gold standard Fungal culture with sputum specimens is shown in table 2 below.

TABle 2. Chest RADIOGRAPH DiAgNOSTIC TEST RESUltS ON FUnGal PNEUMONIA

\begin{tabular}{|c|c|c|c|}
\hline & Fungal culture (+) & Fungal culture (-) & TOTAL \\
\hline Chest x ray (+) & 20 & 64 & 84 \\
\hline Chest x ray (-) & 4 & 22 & 26 \\
\hline TOTAL & 24 & 86 & 110 \\
\hline
\end{tabular}

Chest radiograph sensitivity values were $83.3 \%$ and specificity values was $25.6 \%$.

\subsection{Statistical Analysis and Discussion}

Clinical symptoms Fungal pneumonia is difficult to distinguish from other pneumonia (caused by other microorganisms). According to research conducted by Sukamto, Division of Lung Disease, FK USU., 2004 getting statistical tests with Chi Square clinical symptoms / complaints in the positive group of fungi and negative fungi there were no significant differences. The most common clinical symptoms in Fungal pneumonia and other pneumonia are coughing and followed by shortness of breath, chest pain, fever and other symptoms. This is what causes Fungal pneumonia to be clinically difficult to distinguish from other pneumonia [4]. Determination of Fungal infections in the lung is very difficult because most of the symptoms are initially inconspicuous and often like the symptoms of the common cold or pulmonary infection by other causes. Systemic Fungal symptoms are not typical and can resemble other diseases, it is difficult to distinguish between bacterial pneumonia and Fungal ipneumonia, thus increasing the difficulty of recognizing systemic Fungal pneumonia.

Subjects of the results of positive Fungal sputum culture have a history of long cough (more than 2 weeks), coughing up blood, shortness of breath, chest pain, fever and are usually treated with various antibiotics but do not show improvement [9]. Positive Fungal pneumonia in this study was $21,82 \%$ (24 of the 110 samples clinically leading to Fungal pneumonia). Fungal pneumonia is relatively rare compared to bacterial pneumonia. Diagnosis is usually only enforced if in the clinical course of patients with pneumonia, has been treated with various antibiotics do not show improvement and long-term use of corticosteroids, examination of new Fungal culture / microscopy becomes a thought for advanced therapy $[9,10]$.
Research by Sukamto., 2004 results of sputum culture in SA media in post TB patients with clinical symptoms of pneumonia obtained 22 positive Fungal results from 80 samples (27.5\%). These data indicate that the incidence of Fungal pneumonia compared to other pneumonia is quite rare. This can also be caused by the number of sputum specimens that are irrelevant for examination [4].

Patients with positive Fungal pneumonia in this study were in accordance with risk factors as a trigger for Fungal pneumonia, ie from history there were 4 people with multiple malignancies (Multiple Myeloma) with corticosteroid treatment, 8 people with a history of long cough and long-term antibiotics and others Diabetes Mellitus sufferers, Patients with urinary tract infections with long-term catheter use and Patients with vaginal herpes zooster. In addition to factors over age also have an important role, because usually patients with advanced age (elderly) weakened body defense factors (immunocompromised patients). In this study, over 50 years of age were 7 out of 10 positive people $(70 \%)$ [12,17].

Chest radiograph plays an important role in diagnosing Fungal pneumonia, although sometimes there are some chest radiograph in this Fungal infection similar to tuberculosis or non-specific bacterial infections. Diagnosis Fungal pneumonia is established based on clinical history / symptoms, risk factors, physical examination and investigation. Common diagnostic tests for lung disease include PA and lateral chest radiograph, chest CT Scan and microscopic examination of fungi and sputum culture. The results of this study found varying chest radiograph grouped into positive radiographs (abnormal lesions) $80 \%$, while those negative (normal chest radiograph) $20 \%$. Positive chest radiograph that gave positive sputum culture results were $24(21,82 \%)$. The negative chest radiograph were 13 samples with 4 sputum positive results $(3.64 \%)$. This shows that the chest radiograph examination has been able to show changes in lung tissue in patients with clinical leads to pneumonia, although the abnormalities that occur are still preliminary and the lesions shown are still minimal. While the results of the examination of positive Fungal cultures were smaller than the results of negative Fungal culture examination in a row of $21.82 \%$ and $78.18 \%$. This shows that the incidence of Fungal pneumonia is rare compared to other pneumonia. Examination of positive Fungal cultures if fungi are found in the lungs, where Fungal growth takes a long time.

Chest radiograph sensitivity in this study was $83.3 \%$, which means that the chest radiograph was able to provide a true diagnosis of Fungal pneumonia by $83.3 \%$ of all positive chest radiograph. The specificity of chest radiograph is $25.6 \%$, which means that chest radiograph can completely exclude Fungal pneumonia in only $25.6 \%$ of all samples whose chest radiograph are negative. This high chest radiograph sensitivity can be used as.

\section{CONCLUSION}

The results of this study showed a sensitivity of $83.3 \%$ and specificity of $25.6 \%$. This shows that chest radiograph can be used as a screening examination in patients with clinical Fungal pneumonia but cannot be used as a checkup that can show that patients with clinical Fungal pneumonia are truly healthy not infected with fungi. For the needs of anti-mycotic therapy in 
patients, microbiological sputum examination still needs to be needed.

\section{SUGGESTIONS}

This research is still lacking and needs improvement to be able to do further research, namely:

1. The number of research samples is added so that data validation can be more accurate.

2. Sputum shelter will be better with bronchoscopy, so the growth of microorganisms can be more accurate.

3. The value of sensitivity and specificity of chest radiograph will give better results if the gold standard results of sputum culture on patiouth media to be continued with identification of the cause of fungi.

4. For low chest radiograph specificity values can be corrected by testing the sensitivity and specificity of chest CT Scan.

\section{REFERENCES}

[1] Anwer S, Zafar PMH, Awan H, Zakrya B. Radiological Presentation of Pulmonary Mycosis. Pak JMedRes. 42,no.1. 2003.

[2] Limper AH. The Changing Spectrum of Fungal Infections In Pulmonary and Critical Care Practice: Clinical Approach To Diagnosis. Proc Am Thorac Soc. 2010 May 15;7(3):163-8.

[4] Sukamto. Pemeriksaan Jamur Bilasan Bronkhus pada Penderita Bekas Tuberkulosa Paru. Digit USU Digit Libr 2004. :31.

[5] Erika D Lease, MD. Fungal Diagnostics and Pneumonia. Semin Respir Crit Care Med. 32 (6):663-72.

[6] Bankier AA, Gevenois PA, Sibille Y. Why a series on imaging in the ERJ? Eur Respir J. 2001 Mar 1;17(3):328328 .

[7] Orlowski HLP, McWilliams S, Mellnick VM, Bhalla S, Lubner MG, Pickhardt PJ, et al. Imaging Spectrum of
Invasive Fungal and Fungal-like Infections. RadioGraphics. 2017 Jul;37(4):1119-34.

[8] Ahmad Sarji S, Wan Abdullah W, wastie M. Imaging features of Fungal infection in immuno-suppressed patients in a local ward outbreak. Biomed Imaging Interv J [Internet]. 2006 Apr [cited 2019 May 14];2(2). Available from: http://www.biij.org/2006/2/e21

[9] Widiastuti. The Imaging of Fungal Infection in Lung. Pertemuan Ilmiah Tahunan ke-8 Perhimpunan Dokter Spesialis Radiologi Indonesia; 2012 Feb 24.

[10] Mohammad S Ahmed, MD, MD ER, Khardory N. Respiratory infections in diabetes: Reviewing the risks and challenges. J Respir 2008. June 24;29(7):9.

[11] Beed M, Sherman R, Holden S. Fungal infections and critically ill adults. Contin Educ Anaesth Crit Care Pain. 2014 Dec;14(6):262-7.

[12] Silva RF e. Capítulo 8 - Infecções fúngicas em imunocomprometidos. J Bras Pneumol. 2010 Feb;36(1):142-7.

[13] Franquet T. Imaging of pneumonia: trends and algorithms. Eur Respir J. 2001 Jul 1;18(1):196-208.

[14] Heussel CP. CT Imaging in Aspergillus Lung Infections. $: 54$.

[15] Colen RR, Singer AE, McLoud TC. Cryptococcal Pneumonia in an Immunocompetent Patient. Am J Roentgenol. 2007 Mar;188(3):W281-2.

[16] Guarner J, Brandt ME. Histopathologic Diagnosis of Fungal Infections in the 21st Century. Clin Microbiol Rev. 2011 Apr 1;24(2):247-80.

[17] MD AN, MD RK, MD JL, MD,FACP PB, FACP SJ MD,. Invasive Pulmonary Aspergillosis: Case report and review of literature. J Community Hosp Internial Med Persepectives 2015. 